\title{
Non-gaussianity from the second-order cosmological perturbation
}

\author{
David H. Lyth ${ }^{1, *}$ and Yeinzon Rodríguez ${ }^{1,2, \text { U }}$ \\ ${ }^{1}$ Department of Physics, Lancaster University, Lancaster LA1 4YB, UK \\ ${ }^{2}$ Centro de Investigaciones, Universidad Antonio Nariño, Cll 58A \# 37-94, Bogotá D.C., Colombia
}

(Dated: August 26, 2018)

\begin{abstract}
Several conserved and/or gauge invariant quantities described as the second-order curvature perturbation have been given in the literature. We revisit various scenarios for the generation of second-order non-gaussianity in the primordial curvature perturbation $\zeta$, employing for the first time a unified notation and focusing on the normalisation $f_{\mathrm{NL}}$ of the bispectrum. When $\zeta$ first appears a few Hubble times after horizon exit, $\left|f_{\mathrm{NL}}\right|$ is much less than 1 and is, therefore, negligible. Thereafter $\zeta$ (and hence $f_{\mathrm{NL}}$ ) is conserved as long as the pressure is a unique function of energy density (adiabatic pressure). Non-adiabatic pressure comes presumably only from the effect of fields, other than the one pointing along the inflationary trajectory, which are light during inflation ('light non-inflaton fields'). During single-component inflation $f_{\mathrm{NL}}$ is constant, but multi-component inflation might generate $\left|f_{\mathrm{NL}}\right| \sim 1$ or bigger. Preheating can affect $f_{\mathrm{NL}}$ only in atypical scenarios where it involves light non-inflaton fields. The simplest curvaton scenario typically gives $f_{\mathrm{NL}} \ll-1$ or $f_{\mathrm{NL}}=+5 / 4$. The inhomogeneous reheating scenario can give a wide range of values for $f_{\mathrm{NL}}$. Unless there is a detection, observation can eventually provide a limit $\left|f_{\mathrm{NL}}\right| \lesssim 1$, at which level it will be crucial to calculate the precise observational limit using second order theory.
\end{abstract}

PACS numbers: $98.80 . \mathrm{Cq}$

\section{INTRODUCTION}

Cosmological scales leave the horizon during inflation and re-enter it after Big Bang Nucleosynthesis. Throughout the super-horizon era it is very useful to define a primordial cosmological curvature perturbation, which is conserved if and only if pressure throughout the Universe is a unique function of energy density (the adiabatic pressure condition) [1, 2, 3, 4, 5, 6, 7]. Observation directly constrains the curvature perturbation at the very end of the super-horizon era, a few Hubble times before cosmological scales start to enter the horizon, when it apparently sets the initial condition for the subsequent evolution of all cosmological perturbations. The observed curvature perturbation is almost Gaussian with an almost scale-invariant spectrum.

Cosmological perturbation theory expands the exact equations in powers of the perturbations and keeps terms only up to the $n$th order. Since the observed curvature perturbation is of order $10^{-5}$, one might think that firstorder perturbation theory will be adequate for all comparisons with observation. That may not be the case however, because the PLANCK satellite [8] and its successors may be sensitive to non-gaussianity of the curvature perturbation at the level of second-order perturbation theory [9].

Several authors have treated the non-gaussianity of the primordial curvature perturbation in the context of second-order perturbation theory. They have adopted different definitions of the curvature perturbation and

*Electronic address: d.lyth@lancaster.ac.uk

${ }^{\dagger}$ Electronic address: y.rodriguezgarcia@lancaster.ac.uk obtained results for a variety of situations. In this paper we revisit the calculations, using a single definition of the curvature perturbation which we denote by $\zeta$. In some cases we disagree with the findings of the original authors.

The outline of this paper is the following: in Section we review two definitions of the curvature perturbation found in the literature, which are valid during and after inflation, and establish definite relationships between them; in section [II] we review a third curvature perturbation definition, which applies only during inflation, and study it in models of inflation of the slow-roll variety; in Section IV we describe the present framework for thinking about the origin and evolution of the curvature perturbation; in Section $\nabla$ we see how non-gaussianity is defined and constrained by observation; in Section VI we study the initial non-gaussianity of the curvature perturbation, a few Hubble times after horizon exit; in Section VII we study its subsequent evolution according to some different models. The conclusions are summarised in Section VIII

We shall denote unperturbed quantities by a subscript 0 , and generally work with conformal time $\eta$ defined by the unperturbed line element

$$
d s^{2}=a^{2}(\eta)\left(-d \eta^{2}+\delta_{i j} d x^{i} d x^{j}\right) .
$$

Here $a$ is the scale factor whose present value is taken to be 1 , and a prime denotes $d / d \eta$. Sometimes though we revert to physical time $t$, with a dot meaning $d / d t$ defined by $d / d t \equiv a^{-1} d / d \eta$. We shall adopt the convention that a generic perturbation $g$ is split into a first- and secondorder part according to the formula

$$
g=g_{1}+\frac{1}{2} g_{2} .
$$




\section{TWO DEFINITIONS OF THE CURVATURE PERTURBATION}

\section{A. Preliminaries}

Cosmological perturbations describe small departures of the actual Universe, away from some perfect homogeneous and isotropic universe with the line element Eq. (11). For a generic perturbation it is convenient to make the Fourier expansion

$$
g(\mathbf{x}, \eta)=\frac{1}{(2 \pi)^{3 / 2}} \int d^{3} k g(\mathbf{k}, \eta) e^{i \mathbf{k} \cdot \mathbf{x}},
$$

where the spacetime coordinates are those of the unperturbed Universe. The inverse of the comoving wavenumber, $k^{-1}$, is often referred to as the scale.

A given scale is said to be outside the horizon during the era $a H \gg k$, where $H \equiv \dot{a} / a$ is the Hubble parameter. Except where otherwise stated, our discussion applies only to this super-horizon regime.

When evaluating an observable quantity only a limited range of scales will be involved. The largest scale, relevant for the low multipoles of the Cosmic Microwave Background anisotropy, is $k^{-1} \sim H_{0}^{-1}$ where $H_{0}$ is the present Hubble parameter. The smallest scale usually considered is the one enclosing matter with mass $\sim 10^{6} M_{\odot}$, which corresponds to $k^{-1} \sim 10^{-2} \mathrm{Mpc} \sim$ $10^{-6} H_{0}^{-1}$. The cosmological range of scales therefore extends over only six decades or so.

To define cosmological perturbations in general, one has to introduce in the perturbed Universe a coordinate system $\left(t, x^{i}\right)$, which defines a slicing of spacetime (fixed $t$ ) and a threading (fixed $x^{i}$ ). To define the curvature perturbation it is enough to define the slicing [7].

\section{B. Two definitions of the curvature perturbation}

In this paper we take as our definition of $\zeta$ the following expression for the spatial metric [4, 7, 10, 11, 12, 13, 14] which applies non-perturbatively:

$$
g_{i j}=a^{2}(\eta) \tilde{\gamma}_{i j} e^{2 \zeta} .
$$

Here $\tilde{\gamma}_{i j}$ has unit determinant, and the time-slicing is one of uniform energy density ${ }^{1}$.

It has been shown under weak assumptions 7] that this defines $\zeta$ uniquely, and that $\zeta$ is conserved as long as the pressure is a unique function of energy density. Also, it has been shown that the uniform density slicing

\footnotetext{
${ }^{1}$ It is proved in Ref. 7] that this definition of $\zeta$ coincides with that of Lyth and Wands 15], provided that their slices of uniform coordinate expansion are taken to correspond to those on which the line element has the form Eq. (4) without the factor $e^{2 \zeta}$ (this makes the slices practically flat if $\tilde{\gamma}_{i j} \simeq \delta_{i j}$ ).
}

practically coincides with the comoving slicing (orthogonal to the flow of energy), and with the uniform Hubble slicing (corresponding to uniform proper expansion, that expansion being practically independent of the threading which defines it) [7]. The coincidence of these slicings is important since all three have been invoked by different authors.

Since the matrix $\tilde{\gamma}$ has unit determinant it can be written $\tilde{\gamma}=I e^{h}$, where $I$ is the unit matrix and $h$ is traceless 7]. Assuming that the initial condition is set by inflation, $h$ corresponds to a tensor perturbation (gravitational wave amplitude) which will be negligible unless the scale of inflation is very high. As we shall see later (see footnote 10), the results we are going to present are valid even if $h$ is not negligible, but to simplify the presentation we drop $h$ from the equations. Accordingly, the space part of the metric in the super-horizon regime is supposed to be well approximated by

$$
g_{i j}=a^{2}(\eta) \delta_{i j} e^{2 \zeta} .
$$

At first order, Eq. (5) corresponds to

$$
g_{i j}=a^{2}(\eta) \delta_{i j}(1+2 \zeta) .
$$

Up to a sign, this is the definition of the first-order curvature perturbation adopted by all authors. There is no universally agreed convention for the sign of $\zeta$. Ours coincides with the convention of most of the papers to which we refer, and we have checked carefully that the signs in our own set of equations are correct.

At second order we have

$$
g_{i j}=a^{2}(\eta) \delta_{i j}\left(1+2 \zeta+2 \zeta^{2}\right) .
$$

This is our definition of $\zeta$ at second order.

Malik and Wands [16] instead defined $\zeta$ by Eq. (6) even at second order. Denoting their definition by a subscript MW,

$$
\zeta^{\mathrm{MW}}=\zeta+\zeta^{2}
$$

or equivalently

$$
\zeta_{2}^{\mathrm{MW}}=\zeta_{2}+2\left(\zeta_{1}\right)^{2}
$$

where $\zeta_{1}$ is the first-order quantity whose definition Eq. (6) is agreed by all authors.

To make contact with calculations of the curvature perturbation during inflation, we need some gauge-invariant expressions for the curvature perturbation. 'Gaugeinvariant' means that the definition is valid for any choice of the coordinate system which defines the slicing and threading ${ }^{2}$.

\footnotetext{
${ }^{2}$ In the unperturbed limit the slicing has to be the one on which all quantities are uniform and the the threading has to be orthogonal to it.
} 
We shall write gauge-invariant expressions in terms of $\zeta$ and $\zeta^{\mathrm{MW}}$. First we consider a quantity $\psi^{\mathrm{MW}}$, defined even at second order by

$$
g_{i j}=a^{2}(\eta) \delta_{i j}\left(1-2 \psi^{\mathrm{MW}}\right) .
$$

This definition, which is written in analogy to Eq. (6), applies to a generic slicing. Analogously to Eq. (5) we can consider a quantity $\psi$, valid also in a generic slicing, defined by

$$
g_{i j}=a^{2}(\eta) \delta_{i j} e^{-2 \psi}
$$

On uniform-density slices, $\psi_{1}=\psi_{1}^{\mathrm{MW}}=-\zeta_{1}, \psi_{2}^{\mathrm{MW}}=$ $-\zeta_{2}^{\mathrm{MW}}$, and $\psi_{2}=-\zeta_{2}$. We shall also need the energy density perturbation $\delta \rho$, defined on the generic slicing, as well as the unperturbed energy density $\rho_{0}$.

At first order, the gauge-invariant expression for $\zeta$ has the well-known form

$$
\zeta_{1}=-\psi_{1}-\mathcal{H} \frac{\delta \rho_{1}}{\rho_{0}^{\prime}},
$$

where $\mathcal{H}=a^{\prime} / a$, and the unperturbed energy density satisfies $\rho_{0}^{\prime}=-3 \mathcal{H}\left(\rho_{0}+P_{0}\right)$ with $P_{0}$ being the unperturbed pressure. This expression obviously is correct for the uniform density slicing, and it is correct for all slicings because the changes in the first and second terms induced by a change in the slicing cancel [1, 2, 3, 17, 18].

At second order, Malik and Wands show that [16]

$$
\begin{aligned}
\zeta_{2}^{\mathrm{MW}}= & -\psi_{2}^{\mathrm{MW}}-\mathcal{H} \frac{\delta \rho_{2}}{\rho_{0}^{\prime}}+2 \mathcal{H} \frac{\delta \rho_{1}}{\rho_{0}^{\prime}} \frac{\delta \rho_{1}^{\prime}}{\rho_{0}^{\prime}} \\
& +2 \frac{\delta \rho_{1}}{\rho_{0}^{\prime}}\left(\psi_{1}^{\prime}+2 \mathcal{H} \psi_{1}\right) \\
& -\left(\mathcal{H} \frac{\delta \rho_{1}}{\rho_{0}^{\prime}}\right)^{2}\left(\frac{\rho_{0}^{\prime \prime}}{\mathcal{H} \rho_{0}^{\prime}}-\frac{\mathcal{H}^{\prime}}{\mathcal{H}^{2}}-2\right),
\end{aligned}
$$

which is, again and for the same reason as before, obviously correct for all the slices. Accordingly, from Eq. (9), we can write a gauge invariant definition for our secondorder $\zeta: 3$

$$
\begin{aligned}
\zeta_{2}= & -\psi_{2}-\mathcal{H} \frac{\delta \rho_{2}}{\rho_{0}^{\prime}}+2 \mathcal{H} \frac{\delta \rho_{1}}{\rho_{0}^{\prime}} \frac{\delta \rho_{1}^{\prime}}{\rho_{0}^{\prime}}+2 \frac{\delta \rho_{1}}{\rho_{0}^{\prime}} \psi_{1}^{\prime} \\
& -\left(\mathcal{H} \frac{\delta \rho_{1}}{\rho_{0}^{\prime}}\right)^{2}\left(\frac{\rho_{0}^{\prime \prime}}{\mathcal{H} \rho_{0}^{\prime}}-\frac{\mathcal{H}^{\prime}}{\mathcal{H}^{2}}\right)
\end{aligned}
$$

where the relation

$$
\psi_{2}^{\mathrm{MW}}=\psi_{2}-2\left(\psi_{1}\right)^{2},
$$

coming from Eqs. (10) and (11), has been used.

\footnotetext{
3 This relation has recently been confirmed in Ref. [19] using a nonlinear coordinate-free approach.
}

\section{SLOW-ROLL INFLATION AND A THIRD DEFINITION}

Now we specialize to the era of slow-roll inflation. We consider single-component inflation, during which the curvature perturbation $\zeta$ is conserved, and multicomponent inflation during which it varies. After defining both paradigms, we give a third definition of the curvature perturbation which applies only during inflation.

\section{A. Single-component inflation}

In a single-component inflation model [20, 21] the inflaton trajectory is by definition essentially unique. The inflaton field $\varphi$ parameterises the distance along the inflaton trajectory. In terms of the field variation, slow-roll inflation is characterised by the slow-roll conditions $\epsilon \ll 1$ and $\left|\eta_{V}\right| \ll 1$ [20, 21], where

$$
\begin{aligned}
\epsilon & \equiv-\dot{H} / H^{2}, \\
\eta_{V}-\epsilon & \equiv-\frac{\ddot{\varphi}}{H \dot{\varphi}} .
\end{aligned}
$$

The inflaton field can be taken to be canonically normalised, in which case these definitions are equivalent to conditions on the potential $V$

$$
\begin{aligned}
\epsilon & \equiv \frac{M_{\mathrm{P}}^{2}}{2 V^{2}}\left(\frac{\partial V}{\partial \varphi}\right)^{2}, \\
\eta_{V} & \equiv \frac{M_{\mathrm{P}}^{2}}{V} \frac{\partial^{2} V}{\partial \varphi^{2}},
\end{aligned}
$$

which, together with the slow-roll approximation, lead to the slow-roll behaviour

$$
3 H \dot{\varphi} \approx-\frac{d V}{d \varphi} .
$$

Here $M_{\mathrm{P}}$ is the reduced Planck mass $\left(M_{\mathrm{P}} \equiv\left(8 \pi G_{N}\right)^{-1}\right)$.

Even without the slow-roll approximation, slices of uniform $\varphi$ correspond to comoving slices because a spatial gradient of $\varphi$ would give non-vanishing momentum density. Since comoving slices coincide with slices of uniform energy density, the slices of uniform $\varphi$ coincide also with the latter. Also, since $\varphi$ is a Lorentz scalar, its gauge transformation is the same as that of $\rho$. It follows [22] that we can replace $\rho$ by $\varphi$ in the above expressions:

$$
\begin{aligned}
\zeta_{1}= & -\psi_{1}-\mathcal{H} \frac{\delta \varphi_{1}}{\varphi_{0}^{\prime}} \\
\zeta_{2}^{\mathrm{MW}}= & -\psi_{2}^{\mathrm{MW}}-\mathcal{H} \frac{\delta \varphi_{2}}{\varphi_{0}^{\prime}}+2 \mathcal{H} \frac{\delta \varphi_{1}}{\varphi_{0}^{\prime}} \frac{\delta \varphi_{1}^{\prime}}{\varphi_{0}^{\prime}} \\
& +2 \frac{\delta \varphi_{1}}{\varphi_{0}^{\prime}}\left(\psi_{1}^{\prime}+2 \mathcal{H} \psi_{1}\right) \\
& -\left(\mathcal{H} \frac{\delta \varphi_{1}}{\varphi_{0}^{\prime}}\right)^{2}\left(\frac{\varphi_{0}^{\prime \prime}}{\mathcal{H} \varphi_{0}^{\prime}}-\frac{\mathcal{H}^{\prime}}{\mathcal{H}^{2}}-2\right)
\end{aligned}
$$




$$
\begin{aligned}
\zeta_{2}= & -\psi_{2}-\mathcal{H} \frac{\delta \varphi_{2}}{\varphi_{0}^{\prime}}+2 \mathcal{H} \frac{\delta \varphi_{1}}{\varphi_{0}^{\prime}} \frac{\delta \varphi_{1}^{\prime}}{\varphi_{0}^{\prime}}+2 \frac{\delta \varphi_{1}}{\varphi_{0}^{\prime}} \psi_{1}^{\prime} \\
& -\left(\mathcal{H} \frac{\delta \varphi_{1}}{\varphi_{0}^{\prime}}\right)^{2}\left(\frac{\varphi_{0}^{\prime \prime}}{\mathcal{H} \varphi_{0}^{\prime}}-\frac{\mathcal{H}^{\prime}}{\mathcal{H}^{2}}\right) .
\end{aligned}
$$

\section{B. Multi-component inflation}

Now consider the case of multi-component inflation, where there is a family of inequivalent inflationary trajectories lying in an $\mathrm{N}$-dimensional manifold of field space. If the relevant part of the manifold is not too big it will be a good approximation to take the fields to be canonically normalised. Then the inequivalent trajectories will be curved in field space ${ }^{4}$. To define the trajectories one can choose a fixed basis in field space corresponding to fields $\phi_{1}, \cdots, \phi_{N}$.

Assuming canonical normalisation, multi-component slow-roll inflation is characterised by the conditions

$$
\begin{aligned}
\frac{M_{\mathrm{P}}^{2}}{2 V^{2}}\left(\frac{\partial V}{\partial \phi_{n}}\right)^{2} & \ll 1, \\
\frac{M_{\mathrm{P}}^{2}}{V}\left|\frac{\partial^{2} V}{\partial \phi_{n} \partial \phi_{m}}\right| & \ll 1, \\
3 H \dot{\phi}_{n} & \approx-\frac{\partial V}{\partial \phi_{n}} .
\end{aligned}
$$

The procedure of choosing a fixed basis is quite convenient for calculations, but a different procedure leads to a perhaps simpler theoretical description. This is to take $\varphi$ to parameterise the distance along the inflaton trajectories, just as in single-component inflation, but now with the proviso that uniform $\varphi$ corresponds to uniform field potential (since we work in the slow-roll approximation, this means that the slices in field space of uniform $\varphi$ are orthogonal to the trajectories). Then, in the slow-roll approximation, slices of spacetime with uniform $\varphi$ will again coincide with slices of uniform density (see Fig. 11). Since $\varphi$ is a scalar, Eqs. (21) and (22) will then be valid. This is the simplest form of the gauge-invariant expression, though for a practical calculation it may be better to write it in terms of a fixed basis.

There is a subtlety here. For the first-order case we could define $\varphi$ in a different way; around a given point on the unperturbed trajectory we could choose a fixed field basis, with one of the basis vectors pointing along the trajectory, and define $\varphi$ as the corresponding field component. Then we could choose $\varphi$ to be canonically normalised in the vicinity of the chosen point in field space. That would not work at second order though, because at that order it makes a difference whether $\varphi$ is the appropriate parameterisation of the distance along

\footnotetext{
4 More generally they will be non-geodesics, the geodesics being the trajectories which the background fields could follow if there was no potential term in the scalar Lagrangian [23].
}

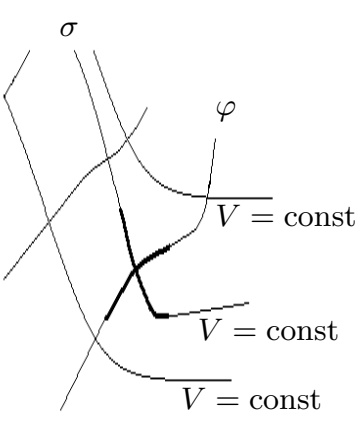

(a)

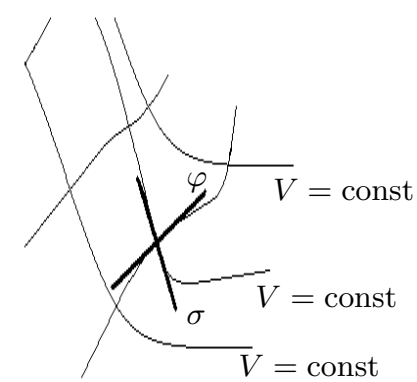

(b)
FIG. 1: Two different procedures for defining the fields in two-component inflation. The fields are denoted by $\varphi$ and $\sigma$. (a) The field $\varphi$ parameterises the distance along the inflaton trajectories, with uniform $\varphi$ corresponding to the equipotential lines. The field $\sigma$ parameterises the distance along the equipotentials. (b) The fields $\varphi$ and $\sigma$ are the components in a fixed orthonormal basis, aligned with the inflationary trajectory at a certain point in field space. The value of $\varphi$ is now the displacement along the tangent vector and the value of $\sigma$ is the displacement along the orthogonal vector. Working to second order in these displacements, the equipotentials no longer coincide with the lines of uniform $\varphi$.

the trajectories (our adopted definition) or the distance along a tangent vector to the trajectory (the alternative definition) (see Fig. 10). Only our adopted one will make Eqs. (22) and (23) valid.

\section{A third definition of the curvature perturbation}

The third definition in the literature applies only during inflation. It was given originally by Acquaviva et. al. 24] for the single-component case, and the generalization to the multi-component case was noted by Rigopoulos 23]. We shall denote this definition by $\zeta^{\mathrm{A}}$.

The definition of Acquaviva et. al. and Rigopoulos is

$$
\begin{aligned}
\zeta_{2}^{\mathrm{A}}= & -\psi_{2}^{\mathrm{MW}}-\mathcal{H} \frac{\delta \varphi_{2}}{\varphi_{0}^{\prime}} \\
& -\frac{\left(\psi_{1}^{\prime}+2 \mathcal{H} \psi_{1}+\mathcal{H} \delta \varphi_{1}^{\prime} / \varphi_{0}^{\prime}\right)^{2}}{\mathcal{H}^{\prime}+2 \mathcal{H}^{2}-\mathcal{H} \varphi_{0}^{\prime \prime} / \varphi_{0}^{\prime}}
\end{aligned}
$$

This is gauge-invariant by construction, with $\varphi$ defined as in Figure 1(a).

It was pointed out by Vernizzi 22] (actually in the context of single-component inflation) that comparing this definition with Eq. (13) gives simply

$$
\zeta_{2}^{\mathrm{A}}=\zeta_{2}^{\mathrm{MW}}-\frac{4 \mathcal{H}^{2}\left(\zeta_{1}\right)^{2}}{\mathcal{H}^{\prime}+2 \mathcal{H}^{2}-\mathcal{H} \varphi^{\prime \prime} / \varphi^{\prime}} .
$$


In the limit of slow-roll the denominator of the last term becomes just $2 \mathcal{H}^{2}$, and then

$$
\zeta_{2}^{\mathrm{A}}=\zeta_{2} .
$$

In other words, this third definition coincides with our adopted one in the slow-roll limit.

Making use of the slow-roll parameters defined in Eqs. (16) and (17), the expression in Eq. (28) gives to firstorder in the slow-roll approximation

$$
\zeta_{2}^{\mathrm{A}}=\zeta_{2}-\left(2 \epsilon-\eta_{V}\right)\left(\zeta_{1}\right)^{2}
$$

\section{THE EVOLUTION OF THE CURVATURE PERTURBATION}

The simplest possibility for the origin of the observed curvature perturbation is that it comes from the vacuum fluctuation of the inflaton field in a single-component model. More recently other possibilities were recognised and we summarise the situation now. Although the discussion is usually applied to the magnitude of the curvature perturbation, it applies equally to the nongaussianity.

\section{A. Heavy, light and ultra-light fields}

On each scale the initial epoch, as far as classical perturbations are concerned, should be taken to be a few Hubble times after horizon exit during inflation. The reason is that all such perturbations are supposed to originate from the vacuum fluctuation of one or more light scalar fields, the fluctuation on each scale being promoted to a classical perturbation around the time of horizon exit.

Considering a fixed basis with canonical normalisation, a light field is roughly speaking one satisfying the flatness condition in Eq. (25). The terminology is suggested by the important special case that the effective potential during inflation is quadratic. Then, a light field is roughly speaking that whose effective mass during inflation is less than the value $H_{*}$ of the Hubble parameter. More precisely, the condition that the vacuum fluctuation be promoted to a classical perturbation is 25]

$$
m<\frac{3}{2} H_{*} .
$$

From now on we focus on the quadratic potential, and take this as the the definition of a light field. Conversely a heavy field may be defined as one for which the condition in Eq. (31) is violated.

During inflation light fields slowly roll according to Eq. (26) (with the vacuum fluctuation superimposed) while the heavy fields presumably are pinned down at an instantaneous minimum of the effective potential. As we have seen, multi-component inflation takes place in a subspace of field space. The fields in this subspace are light, but their effective masses are sufficient to appreciably curve the inflationary trajectories. In the case of both multi-component and single-component inflation, there could also be 'ultra-light' fields, which do not appreciably curve the inflationary trajectory and which therefore have practically no effect on the dynamics of inflation.

\section{B. The evolution of the curvature perturbation}

To describe the behaviour of perturbations during the super-horizon era, without making too many detailed assumptions, it is useful to invoke the separate universe hypothesis 2, 5, 15, 26] after smoothing on a given comoving scale much bigger than the horizon ${ }^{5}$. According to this hypothesis the local evolution at each position is that of some unperturbed universe (separate universe). Of course the separate universe hypothesis can and should be checked where there is a sufficiently detailed model. However, it should be correct on cosmological scales for a very simple reason. The unperturbed Universe may be defined as the one around us, smoothed on a scale a bit bigger than the present Hubble distance. In other words, the separate universe hypothesis is certainly valid when applied to that scale. But the whole range of cosmological scales spans only a few decades. This means that cosmological scales are likely to be huge compared with any scale that is relevant in the early Universe, and accordingly that the separate universe hypothesis should be valid when applied to cosmological scales even though it might fail on much smaller scales (this expectation was verified in a preheating example [27] to which we return later).

We are concerned with the curvature perturbation, which during the super-horizon era is conserved as long as the pressure is a unique function of the energy density (the adiabatic pressure condition). The adiabatic pressure condition will be satisfied if and only if the separate universes are identical (at least as far as the relation between pressure and energy density is concerned) ${ }^{6}$. The condition to have identical universes after a given epoch is that the specification of a single quantity at that epoch is sufficient to determine the entire subsequent evolution.

In the case of single-component inflation, the initial condition may be supplied by the local value of the inflaton field, at the very beginning of the super-horizon

\footnotetext{
${ }^{5}$ When considering linear equations, smoothing is equivalent to dropping short wavelengths fourier components. In the nonlinear situation the smoothing procedure could be in principle ambiguous. In a given situation one should state explicitly which quantities are being smoothed.

${ }^{6}$ Of course the identity will only hold after making an appropriate synchronization of the clocks at different positions. Having made that synchronization, horizon entry will occur at different times in different positions, which can be regarded as the origin of the curvature perturbation.
} 
era when it first becomes classical. Given the separate universe hypothesis, that is the only possibility if the inflaton is the only light field ever to play a significant dynamical role. This means that the curvature perturbation generated at horizon exit during single-component inflation will be equal to the one observed at the approach of horizon entry, provided that the inflaton is the only light field ever to play a dynamical role.

If inflation is multi-component, more than one field is by definition relevant during inflation. Then the curvature perturbation cannot be conserved during inflation. The variation of the curvature perturbation during multicomponent inflation is caused by the vacuum fluctuation orthogonal to the unperturbed inflationary trajectory, which around the time of horizon exit kicks the trajectory onto a nearby one so that the local trajectory becomes position-dependent. After inflation is over, the curvature perturbation will be conserved if the local trajectories lead to practically identical universes. In other words it will be conserved if the light (and ultra-light) fields, orthogonal to the trajectory at the end of inflation, do not affect the subsequent evolution of the Universe.

The curvature perturbation after inflation will vary if some light or ultra-light field, orthogonal to the trajectory at the end of inflation, affects the subsequent evolution of the Universe (to be precise, affects the pressure). As we shall describe in Section VII three types of scenarios have been proposed for this post-inflationary variation of the curvature perturbation.

\section{NON-GAUSSIANITY}

\section{A. Defining the non-gaussianity}

A gaussian perturbation is one whose Fourier components are uncorrelated. All of its statistical properties are defined by its spectrum, and the spectrum $\mathcal{P}_{a}$ of a generic perturbation is conveniently defined $[20,21]$ by $^{7}$

$$
\left\langle g\left(\mathbf{k}_{1}\right) g\left(\mathbf{k}_{2}\right)\right\rangle=\frac{2 \pi^{2}}{k^{3}} \delta^{3}\left(\mathbf{k}_{1}+\mathbf{k}_{2}\right) \mathcal{P}_{g}(k)
$$

the normalisation being chosen so that

$$
\left\langle g^{2}(\mathbf{x})\right\rangle=\int_{0}^{\infty} \mathcal{P}_{g}(k) \frac{d k}{k} .
$$

On cosmological scales a few Hubble times before horizon entry, observation shows that the curvature perturbation is almost Gaussian with $\mathcal{P}_{\zeta}^{1 / 2} \simeq 10^{-5}$.

\footnotetext{
7 Technically the expectation values in this and the following expressions refer to an ensemble of universes but, because the stochastic properties of the perturbations are supposed to be invariant under translations, the expectation values can also be regarded as averages over the location of the observer who defines the origin of coordinates.
}

The simplest kind of non-gaussianity that the curvature perturbation could possess is of the form

$$
\zeta(\mathbf{x})=\zeta_{\mathrm{g}}(\mathbf{x})-\frac{3}{5} f_{\mathrm{NL}}\left(\zeta_{\mathrm{g}}^{2}(\mathbf{x})-\overline{\zeta_{\mathrm{g}}^{2}}\right)
$$

where $\zeta_{\mathrm{g}}$ is Gaussian with $\left\langle\zeta_{\mathrm{g}}\right\rangle=0$, and the non-linearity parameter $f_{\mathrm{NL}}$ is independent of position. We will call this correlated $\chi^{2}$ non-gaussianity. Note that this definition assumes that $\langle\zeta\rangle=0$, which means that the zero Fourier mode (spatial average) is dropped.

Following Maldacena [10], we have inserted the prefactor $-(3 / 5)$ so that in first-order perturbation theory our definition agrees with that of Komatsu and Spergel [9], which is generally the definition people use when comparing theory with observation. Working in firstorder perturbation theory, these authors write $\Phi(\mathbf{x})=$ $\Phi_{g}(\mathbf{x})+f_{\mathrm{NL}}\left(\Phi_{g}^{2}(\mathbf{x})-\overline{\Phi_{g}^{2}}\right)$, and their $\Phi$ is equal to $-3 / 5$ times our $\zeta$.

One of the most powerful observational signatures of non-gaussianity is a nonzero value for the three-point correlator, specified by the bispectrum $B$ defined by $[9,28]$

$\left\langle\zeta\left(\mathbf{k}_{1}\right) \zeta\left(\mathbf{k}_{2}\right) \zeta\left(\mathbf{k}_{3}\right)\right\rangle=(2 \pi)^{-3 / 2} B\left(k_{1}, k_{2}, k_{3}\right) \delta^{3}\left(\mathbf{k}_{1}+\mathbf{k}_{2}+\mathbf{k}_{3}\right)$.

For correlated $\chi^{2}$ non-gaussianity (with the gaussian term dominating)

$$
B\left(k_{1}, k_{2}, k_{3}\right)=-\frac{6}{5} f_{\mathrm{NL}}\left[P_{\zeta}\left(k_{1}\right) P_{\zeta}\left(k_{2}\right)+\text { cyclic }\right],
$$

where $P_{\zeta}(k)=2 \pi^{2} \mathcal{P}_{\zeta}(k) / k^{3}$. For any kind of nonGaussianity one may use the above expression to define a function $f_{\mathrm{NL}}\left(k_{1}, k_{2}, k_{3}\right)$.

Given a calculation of $f_{\mathrm{NL}}$ using first-order perturbation theory, one expects in general that going to second order will change $f_{\mathrm{NL}}$ by an amount of order 1 . On this basis, one expects that a first-order calculation is good enough if it yields $\left|f_{\mathrm{NL}}\right| \gg 1$, but that otherwise a secondorder calculation will be necessary.

The definition Eq. (36) of $f_{\mathrm{NL}}$ is made using our adopted definition of $\zeta$. If $\zeta$ in the definition is replaced by $\zeta^{\mathrm{MW}}$ (with the zero Fourier mode dropped) then $f_{\mathrm{NL}}$ should be replaced by

$$
f_{\mathrm{NL}}^{\mathrm{MW}} \equiv f_{\mathrm{NL}}-\frac{5}{3}
$$

To obtain this expression we used Eq. (8) and dropped terms higher than second order.

All of this assumes that the non-gaussian component of $\zeta$ is fully correlated with the gaussian component. An alternative possibility [29] that will be important for us is if $\zeta$ has the form

$$
\zeta(\mathbf{x})=\zeta_{g}(\mathbf{x})-\frac{3}{5} \tilde{f}_{\mathrm{NL}}\left(\zeta_{\sigma}^{2}(\mathbf{x})-\overline{\zeta_{\sigma}^{2}}\right),
$$

where $\zeta_{g}$ and $\zeta_{\sigma}$ are uncorrelated Gaussian perturbations, normalised to have equal spectra, and the parameter $\tilde{f}_{\mathrm{NL}}$ 
is independent of position. We will call this uncorrelated $\chi^{2}$ non-gaussianity. It can be shown 29] that in this case, $f_{\mathrm{NL}}$ as defined by Eq. (36) is given by

$$
f_{\mathrm{NL}} \sim\left(\frac{\tilde{f}_{\mathrm{NL}}}{1300}\right)^{3} .
$$

\section{B. Observational constraints on the non-gaussianity}

Taking $f_{\mathrm{NL}}$ to denote the non-linearity parameter at the primordial era, let us consider the observational constraints. Detailed calculations have so far been made only with $f_{\mathrm{NL}}$ independent of the wavenumbers, and only by using first-order perturbation theory for the evolution of the cosmological perturbations after horizon entry. It is found [30] that present observation requires $\left|f_{\mathrm{NL}}\right| \lesssim 10^{2}$ making the non-gaussian fraction at most of order $10^{-3}$. The use of first-order perturbation theory in this context is amply justified. Looking to the future though, it is found that the PLANCK satellite will either detect non-gaussianity or reduce the bound to $\left|f_{\mathrm{NL}}\right| \lesssim 5[$,, 28$]$, and that foreseeable future observations can reach a level $\left|f_{\mathrm{NL}}\right| \sim 3[9,28]$.

Although the use of first-order perturbation theory is not really justified for the latter estimates, we can safely conclude that it will be difficult for observation ever to detect a value $\left|f_{\mathrm{NL}}\right| \ll 1$. That is a pity because, as we shall see, such a value is predicted by some theoretical scenarios. On the other hand, other scenarios predict $\left|f_{\mathrm{NL}}\right|$ roughly of order 1. It will therefore be of great interest to have detailed second-order calculations, to establish precisely the level of sensitivity that can be achieved by future observations. A step in this direction has been taken in Refs. 31, 32], where the large-scale cosmic microwave background anisotropy is calculated to second order in terms of only the curvature perturbation (generalizing the first-order Sachs-Wolfe effect [33]).

\section{THE INITIAL NON-GAUSSIANITY}

\section{A. Single-component inflation}

At first order, the curvature perturbation during single-component inflation is Gaussian. Its timeindependent spectrum is given by [20, 21]

$$
\mathcal{P}_{\zeta}(k)=\left[\left(\frac{H}{2 \pi}\right)^{2}\left(\frac{H}{\dot{\varphi}}\right)^{2}\right]_{k=a H},
$$

and its spectral index $n \equiv d \ln \mathcal{P}_{\zeta} / d \ln k$ is given by

$$
n-1=2 \eta_{V}-6 \epsilon .
$$

The spectrum $r$ of the tensor perturbation, defined as a fraction of $\mathcal{P}_{\zeta}$, is also given in terms of the slow-roll parameter $\epsilon$ :

$$
r=16 \epsilon .
$$

If the curvature perturbation does not evolve after single-component inflation is over observation constrains $n$ and $r$, and hence the slow-roll parameters $\eta_{V}$ and $\epsilon$. A current bound [34] is $-0.048<n-1<0.016$ and $r<0.46$. The second bound gives $\epsilon<0.029$, but barring an accurate cancellation the first bound gives $\epsilon \lesssim 0.003$. In most inflation models $\epsilon$ is completely negligible and then the first bound gives $-0.024<\eta_{V}<0.008$ (irrespective of slow-roll inflation models, the upper bound in this expression holds generally, and the lower bound is badly violated only if there is an accurate cancellation). The bottom line of all this is that $\epsilon$ and $\left|\eta_{V}\right|$ are both constrained to be $\lesssim 10^{-2}$.

Going to second order, Maldacena 10 has calculated the bispectrum during single-component inflation (see also Refs. 12, 13, 14, 35, 36, 37, 38]). His result may be written in the form

$$
f_{\mathrm{NL}}=\frac{5}{12}\left(2 \eta_{V}-6 \epsilon-2 \epsilon f\left(k_{1}, k_{2}, k_{3}\right)\right),
$$

with $0 \leq f \leq 5 / 6$. By virtue of the slow-roll conditions, $\left|f_{\mathrm{NL}}\right| \ll 1^{8}$. In other words, the curvature perturbation $\zeta$, as we have defined it, is almost Gaussian during singlecomponent inflation.

From Eq. (30) $\zeta^{\mathrm{A}}$ is also practically gaussian, but this quantity is defined only during inflation and therefore could not be considered as a replacement for $\zeta$. More importantly, $\zeta^{\mathrm{MW}}$ has significant non-gaussianity because, from Eq. (37), it corresponds to $f_{\mathrm{NL}}^{\mathrm{MW}} \approx-5 / 3$.

One may ask why it is our $\zeta$ and not $\zeta^{\mathrm{MW}}$ which is gaussian in the slow-roll limit ${ }^{9}$. One feature that distinguishes our $\zeta$, is that any part of it can be absorbed into the scale factor without altering the rest; indeed

$$
g_{i j}=\delta_{i j} a^{2}(\eta) e^{2 \zeta_{1}+\zeta_{2}}=\delta_{i j} \tilde{a}^{2}(\eta) e^{\zeta_{2}},
$$

with $\tilde{a}=a e^{\zeta_{1}}$ (if we tried to do that with $\zeta_{\mathrm{MW}}$, the part of $\zeta$ not absorbed would have to be re-scaled). This means that an extremely long-wavelength and possible large part of $\zeta$ has no local significance. It also means, in the context of perturbation theory, that the first-order part of $\zeta$ can be absorbed into the scale factor when discussing the second-order part. However, the gaussianity of $\zeta$ does not seem to be related directly to this feature.

\footnotetext{
${ }^{8}$ Near a maximum of the potential 'fast-roll' inflation [39, 40] can take place with $\left|\eta_{V}\right|$ somewhat bigger than 1. Maldacena's calculation does not apply to that case but, presumably, it gives initial non-gaussianity $\left|f_{\mathrm{NL}}\right| \sim 1$. However, the precise initial value of $f_{\mathrm{NL}}$ in this case is not important because the corresponding initial spectral index is far from 1 , which means that the initial curvature perturbation must be negligible.

${ }^{9}$ We thank Paolo Creminelli for enlightening correspondence on this question.
} 
Rather, it has to do with the gauge transformation, relating quantities $\psi_{A}$ and $\psi_{B}$ defined on different slicings.

With our definition [7], the gauge transformation is

$$
\psi_{A}(t, \mathbf{x})-\psi_{B}(t, \mathbf{x})=-\Delta N_{A B}(t, \mathbf{x}),
$$

where $\Delta N_{A B}$ is the number of $e$-folds of expansion going from a slice $B$ to a slice $A$, both of them corresponding to time $t^{10}$. In writing this expression we used physical time $t$ instead of conformal time, the two related by $d t=a d \eta$. Along a comoving worldline, the number of $e$-folds of expansion is defined as $N \equiv \int \tilde{H} d \tau$ where $\tilde{H}$ is the local Hubble parameter and $d \tau$ is the proper time interval [7].

To understand the relevance of this result, take $\psi_{B}=0$ and $\psi_{A}=-\zeta$. The pressure is adiabatic during singlecomponent inflation, which means that $d t$ can be identified with the proper time interval $d \tau$, and the proper expansion rate on slicing $A$ is uniform 7]. As a result, to second order,

$$
\begin{aligned}
\zeta & =H(t) \Delta t(t, \mathbf{x})+\frac{1}{2} \dot{H}(t)(\Delta t(t, \mathbf{x}))^{2} \\
& \simeq H \Delta t(t, \mathbf{x})+\frac{1}{2} \frac{\dot{H}}{H^{2}}(H \Delta t(t, \mathbf{x}))^{2} \\
& \simeq H \Delta t(t, \mathbf{x}) .
\end{aligned}
$$

In the last line we made the slow-roll approximation, and from the second line we can see that the error in $f_{\mathrm{NL}}$ caused by this approximation is precisely $\epsilon$.

We also need the gauge transformation for the inflaton field $\varphi$ in terms of $\Delta t$. Since the slices correspond to the same coordinate time, the unperturbed inflaton field can be taken to be the same on each of them which means that the gauge transformation for $\delta \varphi$ is

$$
\delta \varphi_{A}(t, \mathbf{x})-\delta \varphi_{B}(t, \mathbf{x})=\Delta \varphi_{A B}(t, \mathbf{x}),
$$

where $\Delta \varphi_{A B}$ is the change in $\varphi$ going from slice $B$ to slice $A$. But slice $A$ corresponds to uniform $\varphi$, which means that on slice $B$ to second order

$$
\begin{aligned}
H(t) \frac{\delta \varphi_{B}(t, \mathbf{x})}{\dot{\varphi}_{0}} & =-H(t) \Delta t(t, \mathbf{x})-\frac{1}{2} H(t) \frac{\ddot{\varphi}_{0}}{\dot{\varphi}_{0}}(\Delta t(t, \mathbf{x}))^{2} \\
& \simeq-H \Delta t(t, \mathbf{x})-\frac{1}{2} \frac{\ddot{\varphi}_{0}}{H \dot{\varphi}_{0}}(H \Delta t(t, \mathbf{x}))^{2} \\
& \simeq-H \Delta t(t, \mathbf{x}),
\end{aligned}
$$

where in the last line we used the slow-roll approximation. We can see that the fractional error caused by this approximation is $\ddot{\varphi}_{0} / H \dot{\varphi}_{0}=\epsilon-\eta_{V}$.

Combining Eqs. (46) and (48) we have in the slow-roll approximation

$$
\zeta \simeq-H(t) \frac{\delta \varphi_{B}(t, \mathbf{x})}{\dot{\varphi}_{0}}
$$

10 This expression is valid even when the tensor perturbation is included 7]. As a result, the gauge-invariant expressions mentioned earlier are still valid in that case, as are the results based on them including the present discussion. with fractional error of order $\max \left\{\eta_{V}, \epsilon\right\}$ (this can also be seen directly from Eqs. (21) and (23) evaluated with $\psi=0$, but we give the above argument because it explains why the result is valid for $\zeta$ as opposed to $\zeta^{\mathrm{MW}}$ ).

The final and crucial step is to observe that in the slowroll approximation $\varphi_{B}$ is gaussian, with again a fractional error of order $\max \left\{\eta_{V}, \epsilon\right\}$. This was demonstrated by Maldacena 10] but the basic reason is very simple. The non-gaussianity of $\varphi$ comes either from third and higher derivatives of $V$ (through the field equation in unperturbed spacetime) or else through the back-reaction (the perturbation of spacetime); but the first effect is small [20, 21] by virtue of the flatness requirements on the potential, and the second effect is small because $\dot{\varphi}_{0} / H^{2}$ is small [21]. This explains why $\zeta$ with our adopted definition is practically Gaussian by virtue of the slow-roll approximation.

\section{B. Multi-component inflation}

The flatness and slow-roll conditions Eqs. (24), (25), and (26) ensure that the curvature of the inflationary trajectories is small during the few Hubble times around horizon exit, during which the quantum fluctuation is promoted to a classical perturbation. As a result, the initial curvature perturbation in first-order perturbation theory, is still given by Eq. (40) in terms of the field $\varphi$ that we defined earlier.

What about the initial non-gaussianity generated at second order? In the approximation that the curvature of the trajectories around horizon exit is completely negligible, the orthogonal fields are strictly massless. Such fields would not affect Maldacena's second-order calculation, which would therefore still give the initial nongaussianity. It is not quite clear whether the curvature can really be neglected, as it can be in the first-order case, and it may therefore be that the initial non-gaussianity in multi-field inflation is different from Maldacena's result. Even if that is the case though, we can safely say that the initial non-gaussianity corresponds to $\left|f_{\mathrm{NL}}\right| \ll 1$, since the curvature of the trajectories is certainly small.

\section{THE EVOLUTION AFTER HORIZON EXIT}

\section{A. Single-component inflation and $\zeta_{2}^{\mathrm{A}}$}

During single-component inflation the curvature perturbation $\zeta$, as we have defined it, does not evolve. From its definition Eq. (8), the same is true of $\zeta_{2}^{\mathrm{MW}}$.

In contrast $\zeta_{2}^{\mathrm{A}}$, given by Eq. (30), will have the slow variation 22

$$
\dot{\zeta}_{2}^{\mathrm{A}} \approx-\left(2 \dot{\epsilon}-\dot{\eta}_{V}\right)\left(\zeta_{1}\right)^{2} .
$$

This variation has no physical significance, being an artifact of the definition. 
Using a particular gauge, Acquaviva et. al. 24] have calculated $\dot{\zeta}_{2}^{\mathrm{A}}$ in terms of first-order quantities $\psi_{1}, \delta \varphi_{1}$, and their derivatives, and they have displayed the result as an indefinite integral

$$
\zeta_{2}^{\mathrm{A}}(t)=\int^{t} A(t) d t+B(t)
$$

Inserting an initial condition, valid a few Hubble times after horizon exit, this becomes

$$
\zeta_{2}^{\mathrm{A}}(t)=\zeta_{2}^{\mathrm{A}}\left(t_{\mathrm{i}}\right)+\int_{t_{\mathrm{i}}}^{t} A(t) d t+\left.B\right|_{t_{\mathrm{i}}} ^{t} .
$$

In view of our discussion, it is clear that these equations will, if correctly evaluated, just reproduce the time dependence of Eq. (501).

The authors of Ref. 24] also present an equation for $\dot{\zeta}_{2}^{\mathrm{A}}$, again involving only first-order quantities, which is valid also before horizon entry. Contrary to the claim of the authors, this classical equation cannot by itself be used to calculate the initial value (more precisely, the stochastic properties of the initial value) of $\zeta_{2}^{\mathrm{A}}$. In particular, it cannot by itself reproduce Maldacena's calculation of the bispectrum.

It is true of course that in the Heisenberg picture the quantum operators satisfy the classical field equations. In first-order perturbation theory, where the equations are linear, this allows one to calculate the curvature perturbation without going to the trouble of calculating the second-order action 21] (at the $n$th order of perturbation theory the action has to be evaluated to order $n+1$ if it is to be used). At second order in perturbation theory it remains to be seen whether the Heisenberg picture can provide a useful alternative to Maldacena's calculation, who adopted the interaction picture and calculated the action to third order.

\section{B. Multi-component inflation}

During multi-component inflation the curvature perturbation by definition varies significantly along a generic trajectory, which means that non-gaussianity is generated at some level. So far only a limited range of models has been investigated [41, 42, 43, 44, 45, 46]. To keep the spectral tilt within observational bounds, the unperturbed trajectory in these models has to be specially chosen, but the choice might be justified by a suitable initial condition.

We shall consider here a calculation by Enqvist and Väihkönen in Ref. 45]. Following the same line as Acquaviva et. al. [24], they study a two-component inflation model, in which the only important parts of the potential are

$$
V(\varphi, \sigma)=V_{0}+\frac{1}{2} m_{\sigma}^{2} \sigma^{2}+\frac{1}{2} m_{\varphi}^{2} \varphi^{2} .
$$

The masses are both supposed to be less than $(3 / 2) H_{*}$, so that this is a two-component inflation model, and the above form of the potential is supposed to hold for some number $\Delta N$ of $e$-folds after cosmological scales leave the horizon. They take the unperturbed inflation trajectory to have $\sigma_{0}=0$, and the idea is to calculate the amount of non-gaussianity generated after $\Delta N e$-folds. Irrespective of any later evolution, this calculated nongaussianity will represent the minimal observed one (unless non-gaussianity generated later happens to cancel it).

It is supposed that the condition $\sigma_{0}=0$, as well as the ending of inflation, will come from a tree-level hybrid potential,

$$
V(\varphi, \sigma)=V_{0}-\frac{1}{2} m_{0}^{2} \sigma^{2}+\frac{1}{4} \lambda \sigma^{4}+\frac{1}{2} m_{\varphi}^{2} \varphi^{2}+\frac{1}{2} g^{2} \sigma^{2} \varphi^{2} .
$$

Like the original authors though, we shall not investigate the extent to which Eq. (54) can reproduce Eq. (153) for at least some number of $e$-folds. We just focus on Eq. (53), with the assumption $\sigma_{0}=0$ for the unperturbed trajectory.

Because $\sigma_{0}=0$, the unperturbed trajectory is straight, and at first order the curvature perturbation $\zeta$ is conserved. This is not the case though at second order. Adopting the definition $\zeta^{\mathrm{A}}$, the authors of 45] give an expression for $\zeta_{2}^{\mathrm{A}}$ similar to that in Eq. (52) describing the evolution of the second-order curvature perturbation on superhorizon scales ${ }^{11}$. This equation, in the generalized longitudinal gauge, reads (from Eq. (67) in Ref. [45]):

$$
\begin{aligned}
& \zeta_{2}^{\mathrm{A}}(t)-\zeta_{2}^{\mathrm{A}}\left(t_{\mathrm{i}}\right)= \\
& -\frac{1}{\epsilon H M_{\mathrm{P}}^{2}}\left\{\int _ { t _ { \mathrm { i } } } ^ { t } \left[6 H \Delta^{-2} \partial_{i}\left(\delta \dot{\sigma}_{1} \partial^{i} \delta \sigma_{1}\right)-2\left(\delta \dot{\sigma}_{1}\right)^{2}\right.\right. \\
& +4 \Delta^{-2} \partial_{i}\left(\delta \dot{\sigma}_{1} \partial^{i} \delta \sigma_{1}\right)^{\cdot}+m_{\sigma}^{2}\left(\delta \sigma_{1}\right)^{2} \\
& +\left(\epsilon-\eta_{V}\right) 6 H \Delta^{-4} \partial_{i}\left(\partial_{k} \partial^{k} \delta \sigma_{1} \partial^{i} \delta \sigma_{1}\right)^{\cdot} \\
& +\left(\epsilon-\eta_{V}\right) H \Delta^{-4} \partial_{i} \partial^{i}\left(\partial_{k} \delta \sigma_{1} \partial^{k} \delta \sigma_{1}\right)^{\cdot} \\
& -3 \Delta^{-4} \partial_{i}\left(\partial_{k} \partial^{k} \delta \sigma_{1} \partial^{i} \delta \sigma_{1}\right)^{\cdot \cdot} \\
& \left.-\frac{1}{2} \Delta^{-4} \partial_{i} \partial^{i}\left(\partial_{k} \delta \sigma_{1} \partial^{k} \delta \sigma_{1}\right)^{\cdot \cdot}\right] d t \\
& +\left[-\Delta^{-2} \partial_{i}\left(\delta \dot{\sigma}_{1} \partial^{i} \delta \sigma_{1}\right)+3 \Delta^{-4} \partial_{i}\left(\partial_{k} \partial^{k} \delta \sigma_{1} \partial^{i} \delta \sigma_{1}\right)^{\cdot}\right. \\
& +\frac{1}{2} \Delta^{-4} \partial_{i} \partial^{i}\left(\partial_{k} \delta \sigma_{1} \partial^{k} \delta \sigma_{1}\right)^{\cdot} \\
& +3 \epsilon H \Delta^{-4} \partial_{i}\left(\partial_{k} \partial^{k} \delta \sigma_{1} \partial^{i} \delta \sigma_{1}\right) \\
& \left.\left.+\frac{\epsilon H}{2} \Delta^{-4} \partial_{i} \partial^{i}\left(\partial_{k} \delta \sigma_{1} \partial^{k} \delta \sigma_{1}\right)\right]\left.\right|_{t_{\mathrm{i}}} ^{t}\right\}
\end{aligned}
$$

11 The fields $\varphi$ and $\sigma$ in Eq. 53 are supposed to be canonically normalised, which means that $\varphi$ is not the field appearing in the Rigopoulos definition Eq. 27 of $\zeta^{\mathrm{A}}$. Instead the authors of [45] give an equivalent definition in terms of the canonically normalised fields. 
where $\Delta^{-2}$ is the inverse of the Laplacian operator.

Assuming that this expression is correct, we consider the non-gaussianity it may generate. Following the original authors, we note first that

$$
\left|H \frac{\delta \sigma_{1}}{\dot{\varphi}_{0}}\right| \sim\left|H \frac{\delta \varphi_{1}}{\dot{\varphi}_{0}}\right|=\left|\zeta_{1}\right| \equiv \text { constant },
$$

which is a good approximation since the first-order perturbation equation of the effectively massless field $\sigma$ is the same as the first-order perturbation equation of the inflaton field $\varphi$ on superhorizon scales. Moreover, the time derivative of the first-order perturbation in $\sigma$ can be estimated as

$$
\left|\delta \dot{\sigma}_{1}\right| \sim \frac{m_{\sigma}^{2}}{H}\left|\delta \sigma_{1}\right|
$$

assuming slow-roll conditions. If we also assume that $H$ and $m_{\sigma}^{2}$ are almost constants in time, we end up with

$$
\begin{aligned}
\zeta_{2}^{\mathrm{A}}(t)-\zeta_{2}^{\mathrm{A}}\left(t_{\mathrm{i}}\right)= & -\frac{1}{\epsilon H M_{\mathrm{P}}^{2}} \int_{t_{\mathrm{i}}}^{t}\left[6 H \Delta^{-2} \partial_{i}\left(\delta \dot{\sigma}_{1} \partial^{i} \delta \sigma_{1}\right)\right. \\
& \left.-2\left(\delta \dot{\sigma}_{1}\right)^{2}+m_{\sigma}^{2}\left(\delta \sigma_{1}\right)^{2}\right] d t
\end{aligned}
$$

For $m^{2} / H^{2} \ll 1$ the first and third term in the integrand dominate, whereas all the three terms become of the same order of magnitude for $m^{2} / H^{2} \sim 1$ which is the limit of applicability of the calculation. In any case, the typical magnitude of the right-hand side is of order

$$
\Delta N \frac{m_{\sigma}^{2}}{H^{2}}\left|\zeta_{1}\right|^{2}
$$

with $\Delta N$ the number of e-folds specified by the integral limits. This looks big, but we have to remember that the right hand side is uncorrelated with the inflaton perturbation $\delta \phi$ which generates $\zeta_{1}^{\mathrm{A}}$. Therefore, Eq. (38) as opposed to Eq. (34) applies, and we would need $\Delta N \sim 1300$ to get even $f_{\mathrm{NL}} \sim 1$, which is impossible.

These conclusions differ sharply from those of Enqvist and Väihkönen [45] who actually find $\zeta_{2}^{A} \propto$ $\mathcal{O}\left(\epsilon, \eta_{V}, m_{\sigma}^{2} / H^{2}\right)\left(\zeta_{1}\right)^{2}$. ${ }^{12}$ Their estimate of the righthand side of Eq. (58) does not contain our factor $\Delta N$, but much more importantly they estimate $f_{\mathrm{NL}}$ as if the right hand side were fully correlated with $\zeta_{1}^{\mathrm{A}}$ to conclude that the model can give $f_{\mathrm{NL}} \sim 1$.

The reason of the discrepancy lies in the Eq. (71) in Ref. [45], which we write in the same form as our Eq. (59):

$$
\zeta_{2}^{\mathrm{A}}(t)-\zeta_{2}^{\mathrm{A}}\left(t_{\mathrm{i}}\right)=-\frac{1}{\epsilon H M_{\mathrm{P}}^{2}} \int_{t_{\mathrm{i}}}^{t}\left[6 H \Delta^{-2} \partial_{i}\left(\delta \dot{\sigma}_{1} \partial^{i} \delta \sigma_{1}\right)\right.
$$

12 In the proper treatment of the integral and its initial condition the factors $\epsilon\left(\zeta_{1}\right)^{2}, \eta_{V}\left(\zeta_{1}\right)^{2}$, and $m_{\sigma}^{2} / H^{2}\left(\zeta_{1}\right)^{2}$ cancel out since the evaluated quantity is in this case $\zeta_{2}^{\mathrm{A}}(t)-\zeta_{2}^{\mathrm{A}}\left(t_{\mathrm{i}}\right)$, and $\zeta_{1}$ is conserved. This was not taken into account in Ref. [45].

$$
\begin{aligned}
& \left.-2\left(\delta \dot{\sigma}_{1}\right)^{2}+m_{\sigma}^{2}\left(\delta \sigma_{1}\right)^{2}\right] d t \\
= & -\frac{1}{\epsilon H M_{\mathrm{P}}^{2}} \int_{t_{\mathrm{i}}}^{t}\left[-2 \Delta^{-2} \partial_{i}\left(\delta \ddot{\sigma}_{1} \partial^{i} \delta \sigma_{1}\right)\right. \\
& \left.-2\left(\delta \dot{\sigma}_{1}\right)^{2}\right],
\end{aligned}
$$

where the equation of motion $\delta \ddot{\sigma}_{1}+3 H \delta \dot{\sigma}_{1}+m_{\sigma}^{2} \delta \sigma_{1}=0$ has been used. Enqvist and Väihkönen seem to have neglected the factor $\delta \ddot{\sigma}_{1}$ in the above expression, keeping only the term $-2\left(\delta \dot{\sigma}_{1}\right)^{2}$ in the integrand, which gives the partial estimate

$$
\zeta_{2}^{\mathrm{A}}(t)-\zeta_{2}^{\mathrm{A}}\left(t_{\mathrm{i}}\right) \sim \Delta N \frac{m_{\sigma}^{4}}{H^{4}}\left|\zeta_{1}\right|^{2}<<\frac{m_{\sigma}^{2}}{H^{2}}\left|\zeta_{1}\right|^{2} .
$$

This last step is wrong because $\delta \ddot{\sigma}_{1}$ can only be neglected compared with $3 H \delta \dot{\sigma}_{1}+m_{\sigma}^{2} \delta \sigma_{1}$, which is not present in the integrand in Eq. (60). Moreover, what we need is the integral of $\delta \ddot{\sigma}_{1}$, i.e. $\delta \dot{\sigma}_{1}$, which is in any case nonnegligible. That is the origin of our new term

$$
\Delta N \frac{m_{\sigma}^{2}}{H^{2}}\left|\zeta_{1}\right|^{2}
$$

in Eq. (59). As a conclusion the level of non-gaussianity in this hybrid-type model seems to be much smaller than previously thought as the condition $m_{\sigma} \lesssim H$ is presumably not satisfied for a sufficient number of e-folds.

\section{Preheating}

Now we turn to the possibility that significant nongaussianity could be generated during preheating. Preheating is the term used to describe the energy loss by scalar fields which might occur between the end of inflation and reheating 47, 48], the latter being taken to correspond to the decay of individual particles which leads to more or less complete thermalisation of the Universe. Preheating typically produces marginally-relativistic particles, which decay before reheating.

It was suggested a long time ago [49, 50] that preheating might cause the cosmological curvature perturbation to vary at the level of first-order perturbation theory, perhaps providing its main origin. More recently it has been suggested [46, 51, 52 that preheating might cause the curvature perturbation to vary at second order, providing the main source of its non-gaussianity.

If the separate universe hypothesis is correct, a variation of the curvature perturbation during preheating can occur only in models of preheating which contain a noninflaton field that is light during inflation. This is not the case for the usual preheating models that were considered in 46, 51, 52], and accordingly one does not expect that significant non-gaussianity will be generated in those models ${ }^{13}$. This is not in conflict with the findings

13 The preheating model considered in [46] contains a field which 
of $46,51,52$ because the curvature perturbation is not actually considered there. Instead the perturbation $\psi^{\mathrm{MW}}$ in the longitudinal gauge is considered, which is only indirectly related to $\zeta$ by Eqs. (8), (12) and (13) ${ }^{14}$. We conjecture that non-gaussianity for the curvature perturbation on cosmological scales is not generated in the usual preheating models, but that instead the curvature perturbation remains constant on cosmological scales. This should of course be checked, in the same spirit that the constancy of the curvature perturbation was checked at the first-order level [27].

The situation is different for preheating models which contain a non-inflaton field that is light during inflation. At least three types of models have been proposed with that feature [46, 53, 54, 55]. Except for 46] only the magnitude of the curvature perturbation has been considered, but in all three cases it might be that significant non-gaussianity is also generated.

\section{The curvaton scenario}

In the simplest version of the curvaton scenario 56, 57], the curvaton field $\sigma$ is ultra-light during inflation and has no significant evolution until it starts to oscillate during some radiation-dominated era. Until this oscillation gets under way, the curvature perturbation is supposed to be negligible (compared with its final observed value). The potential during the oscillation is taken to be quadratic, which will be a good approximation after a few Hubble times even if it fails initially. The curvature perturbation is generated during the oscillation, and is supposed to be conserved after the curvaton decays. Here we give a generally-valid formula for the non-gaussianity in the curvaton scenario, extending somewhat the earlier calculations.

The local energy density $\rho_{\sigma}$ of the curvaton field is given by

$$
\rho_{\sigma}(\eta, \mathbf{x}) \approx \frac{1}{2} m_{\sigma}^{2} \sigma_{a}^{2}(\eta, \mathbf{x})
$$

where $\sigma_{a}(\eta, \mathbf{x})$ represents the amplitude of the oscillations and $m_{\sigma}$ is the effective mass. It is proportional to $a(\eta, \mathbf{x})^{-3}$ where $a$ is the locally-defined scale factor. This means that the perturbation $\delta \rho_{\sigma} / \rho_{\sigma}$ is conserved if the slicing is chosen so that the expansion going from one slicing to the next is uniform [15]. The flat slicing corresponding to $\psi^{\mathrm{MW}}=0$ has this property [7, 15] and accordingly $\delta \rho_{\sigma}$ is defined on that slicing.

Assuming that the fractional perturbation is small (which we shall see is demanded by observation) it is

may be heavy or light; we refer here to the part of the calculation that considers the former case.

14 The slices of the longitudinal gauge are orthogonal to the threads of zero shear, and $\psi^{\mathrm{MW}}$ on them is very different from the curvature perturbation $\zeta$. given by

$$
\frac{\delta \rho_{\sigma}}{\rho_{\sigma}}=2 \frac{\delta \sigma_{a}}{\sigma_{a}}+\left(\frac{\delta \sigma_{a}}{\sigma_{a}}\right)^{2} .
$$

We first assume that $\sigma(\mathbf{x})$ has no evolution between inflation and the onset of oscillation. Then $\delta \sigma_{a} / \sigma_{a}$ will be equal to its value just after horizon exit, which we saw earlier will be practically gaussian.

The total density perturbation is given by

$$
\frac{\delta \rho}{\rho}=\Omega_{\sigma} \frac{\delta \rho_{\sigma}}{\rho_{\sigma}},
$$

where $\Omega_{\sigma} \equiv \rho_{\sigma} / \rho \propto a$ is the fraction of energy density contributed by the curvaton. Adopting the sudden-decay approximation, the constant curvature perturbation obtaining after the curvaton decays is given by Eqs. (12) and (14), evaluated just before curvaton decay and with $\psi=0$. In performing that calculation, the exact expression Eq. (64) can, without loss of generality, be identified with the first-order part $\delta \rho_{\sigma_{1}} / \rho_{\sigma_{0}}$, the second- and higher-order parts being set at zero.

Adopting the first-order curvature perturbation in Eq. (12), one finds [57] chi-squared non-gaussianity coming from the second term of Eq. (64),

$$
f_{\mathrm{NL}}=-\frac{5}{4 r}
$$

with

$$
r \equiv \frac{3 \rho_{\sigma}}{4 \rho_{r}+3 \rho_{\sigma}}
$$

evaluated just before decay $\left(\rho_{r}\right.$ is the radiation density). Going to the second-order expression one finds [58] additional chi-squared non-gaussianity. The final nonlinearity parameter $f_{\mathrm{NL}}=f_{\mathrm{NL}}^{\mathrm{MW}}+5 / 3$ is given by

$$
f_{N L}=\frac{5}{3}+\frac{5}{6} r-\frac{5}{4 r}
$$

If $\Omega_{\sigma} \ll 1$ then $f_{\mathrm{NL}}$ is strongly negative and the present bound on it requires $\Omega_{\sigma} \gtrsim 0.01$ (combined with the typical value $\zeta \sim 10^{-5}$, this requires $\delta \rho_{\sigma} / \rho_{\sigma} \ll 1$ as advertised). If instead $\Omega_{\sigma}=1$ to good accuracy, then $f_{\mathrm{NL}}=+5 / 4$. Either of these possibilities may be regarded as generic whereas the intermediate possibility $\left(\left|f_{\mathrm{NL}}\right| \sim 1\right.$ but $\left.f_{\mathrm{NL}} \neq 5 / 4\right)$ requires a special value of $\Omega_{\sigma}$ just a bit less than 1 .

Finally, we consider the case that $\sigma$ evolves between horizon exit and the era when the sinusoidal oscillation begins. If $\sigma_{a}$ (the amplitude of oscillation at the latter era) is some function $g\left(\sigma_{*}\right)$ of the value a few Hubble times after horizon exit, then

$$
\delta \sigma_{a}=g^{\prime} \delta \sigma_{*}+\frac{1}{2} g^{\prime \prime}\left(\delta \sigma_{*}\right)^{2},
$$

where the prime means derivative with respect to $\sigma_{*}$. Repeating the above calculation one finds

$$
f_{\mathrm{NL}}=\frac{5}{3}+\frac{5}{6} r-\frac{5}{4 r}\left(1+\frac{g g^{\prime \prime}}{g^{\prime 2}}\right) .
$$


The final term is the first-order result (given originally in 59]), the middle term is the second-order correction found in [58], and the first term converts from $f_{\mathrm{NL}}^{\mathrm{MW}}$ to $f_{\mathrm{NL}}$.

\section{E. The inhomogeneous reheating scenario}

The final scenario that has been suggested for the origin of the curvature perturbation is its generation during some spatially inhomogeneous reheating process 60, 61, 62, 63. . Before a reheating process the cosmic fluid is dominated by matter (non-relativistic particles, or small scalar field oscillations which are equivalent to particles) which then decay into thermalised radiation. At least one reheating process, presumably, has to occur to give the initial condition for Big Bang Nucleosynthesis, but there might be more than one.

The inhomogeneous reheating scenario in its simplest form supposes that the curvature perturbation is negligible before the relevant reheating process, and constant afterwards. The inhomogeneous reheating corresponds to a spatially varying value (a perturbation) of the local Hubble parameter $H_{\text {reh }}(\mathbf{x})$ at the decay epoch (or equivalently of the local energy density), and this generates the final curvature perturbation. The perturbation in $H_{\text {reh }}$ occurs presumably because it depends on the value of some non-inflaton 'modulon' field $\chi$ which was light or ultra-light during inflation.

In contrast with the curvaton scenario, where the form $\rho_{\sigma}$ can reasonably be taken as $\rho_{\sigma} \propto \sigma^{2}$, the inhomogeneous reheating scenario does not suggest any particular form for $H_{\text {reh }}(\chi)$. Depending on the form, the inhomogeneous reheating scenario presumably can produce a wide range of values for $f_{\mathrm{NL}}$.

\section{CONCLUSIONS}

We have examined a number of scenarios for the production of a non-gaussian primordial curvature perturbation, presenting the results with a unified notation. These are the single-component inflation, multi-component inflation, preheating, curvaton, and inhomogeneous reheating scenarios. Although the trispectrum may give a competitive observational signal [64, 65], we have focused only on the bispectrum which is characterised by the parameter $f_{\mathrm{NL}}$. In all cases our treatment is based on existing ones, though we do not always agree with the original authors.

The preheating and inhomogeneous reheating scenarios cover a range of possibilities, which have not been fully explored but which can presumably allow a wide range for $f_{\mathrm{NL}}$. The same is true of multi-component inflation, except that extremely large values comparable with the current bound $\left|f_{\mathrm{NL}}\right| \lesssim 10^{2}$ seem relatively unlikely. In contrast, the simplest curvaton scenario can produce a strongly negative value (even violating the current bound). However, in the important special case where the curvaton dominates the energy density before it decays, it gives precisely $f_{\mathrm{NL}}=+5 / 4$. Finally, for the single-component inflation case, Maldacena's calculation combined with current constraints on the spectral tilt show that it has magnitude less than $10^{-2}$. These result are summarised in the Table $\square$

TABLE I: Non-gaussianity according to different scenarios for the creation of the curvature perturbation. For the simplest curvaton scenario, $f_{\mathrm{NL}}=+5 / 4$ is a favoured value.

\begin{tabular}{|c|c|c|c|c|}
\hline \multirow{3}{*}{$\begin{array}{l}\text { Scenario } \\
\text { Single-component }\end{array}$} & $\left|f_{\mathrm{NL}}\right| \ll 1$ & \multicolumn{3}{|c|}{$\left|f_{\mathrm{NL}}\right| \simeq 1 f_{\mathrm{NL}} \ll-1 f_{\mathrm{NL}} \gg 1$} \\
\hline & & & & \\
\hline & yes & no & no & no \\
\hline $\begin{array}{l}\text { Multi-component } \\
\text { inflation }\end{array}$ & likely & possible & possible & possible \\
\hline $\begin{array}{l}\text { Simplest } \\
\text { curvaton scenario }\end{array}$ & unlikely & likely & likely & no \\
\hline
\end{tabular}

In the near future, results from the Wilkinson Microwave Anisotropy Probe (WMAP) 66] or elsewhere may detect a value $\left|f_{\mathrm{NL}}\right| \gg 1$. If that does not happen, then PLANCK [8] or a successor will either detect a value $\left|f_{\mathrm{NL}}\right| \sim 1$, or place a bound $\left|f_{\mathrm{NL}}\right| \lesssim 1$. The precise level at which this will be possible has yet to be determined because it would require a second-order calculation of all relevant observational signatures. The example of the simplest curvaton scenario, where $f_{\mathrm{NL}}=+5 / 4$ is a favoured value, shows that such a calculation and the eventual observations will be well worthwhile.

\section{Acknowledgments}

We thank Karim A. Malik for discussions on all aspects of this work, and Kari Enqvist, Asko Jokinen, Anupam Mazumdar, Tuomas Multamäki, and Antti Väihkönen for a clarification of their work. We also thank Misao Sasaki and Paolo Creminelli for discussions. D.H.L. is supported by PPARC grants PPA/G/O/2002/00469, PPA/V/S/2003/00104, $\mathrm{PPA} / \mathrm{G} / \mathrm{S} / 2002 / 00098$ and $\mathrm{PPA} / \mathrm{Y} / \mathrm{S} / 2002 / 00272$, and by European Union grant MRTN-CT-2004-503369. Y.R. is fully supported by COLCIENCIAS (COLOMBIA), and partially supported by COLFUTURO (COLOMBIA), UNIVERSITIES UK (UK), and the Department of Physics of Lancaster University (UK). 
[1] J. M. Bardeen, Phys. Rev. D 22, 1882 (1980).

[2] J. M. Bardeen, P. J. Steinhardt, and M. S. Turner, Phys. Rev. D 28, 679 (1983).

[3] D. H. Lyth, Phys. Rev. D 31, 1792 (1985).

[4] D. S. Salopek and J. R. Bond, Phys. Rev. D 42, 3936 (1990).

[5] D. Wands, K. A. Malik, D. H. Lyth, and A. R. Liddle, Phys. Rev. D 62, 043527 (2000).

[6] G. I. Rigopoulos and E. P. S. Shellard, Phys. Rev. D 68, 123518 (2003).

[7] D. H. Lyth, K. A. Malik, and M. Sasaki, JCAP 0505, 004 (2005).

[8] ESA's PLANCK mission homepage: http://planck. esa.int/.

[9] E. Komatsu and D. N. Spergel, Phys. Rev. D 63, 063002 (2001)

[10] J. Maldacena, JHEP 0305, 013 (2003).

[11] P. Creminelli and M. Zaldarriaga, JCAP 0410, 006 (2004).

[12] D. Seery and J. E. Lidsey, astro-ph/0503692

[13] G. I. Rigopoulos, E. P. S. Shellard, and B. J. W. van Tent, astro-ph/0410486

[14] G. Calcagni, astro-ph/0411773

[15] D. H. Lyth and D. Wands, Phys. Rev. D 68, 103515 (2003).

[16] K. A. Malik and D. Wands, Class. Quantum Grav. 21, L65 (2004).

[17] S. Matarrese, S. Mollerach, and M. Bruni, Phys. Rev. D 58, 043504 (1998).

[18] M. Bruni, S. Matarrese, S. Mollerach, and S. Sonego, Class. Quantum Grav. 14, 2585 (1997).

[19] D. Langlois and F. Vernizzi, astro-ph/0503416

[20] D. H. Lyth and A. Riotto, Phys. Rep. 314, 1 (1999).

[21] A. R. Liddle and D. H. Lyth, Cosmological inflation and large-scale structure, Cambridge University Press, 2000.

[22] F. Vernizzi, Phys. Rev. D 71, 061301(R) (2005).

[23] G. Rigopoulos, Class. Quantum Grav. 21, 1737 (2004).

[24] V. Acquaviva, N. Bartolo, S. Matarrese, and A. Riotto, Nucl. Phys. B 667, 119 (2003).

[25] M. Mijic, Phys. Rev. D 57, 2138 (1998).

[26] M. Sasaki and T. Tanaka, Prog. Theor. Phys. 99, 763 (1998).

[27] A. R. Liddle, D. H. Lyth, K. A. Malik, and D. Wands, Phys. Rev. D 61, 103509 (2000).

[28] N. Bartolo, E. Komatsu, S. Matarrese, and A. Riotto, Phys. Rep. 402, 103 (2004).

[29] L. Boubekeur and D. H. Lyth, astro-ph/0504046

[30] E. Komatsu et. al., Astrophys. J. Suppl. Ser. 148, 119 (2003).

[31] N. Bartolo, S. Matarrese, and A. Riotto, Phys. Rev. Lett. 93, 231301 (2004).

[32] P. Creminelli and M. Zaldarriaga, Phys. Rev. D 70, 083532 (2004).

[33] R. K. Sachs and A. M. Wolfe, Astrophys. J. 147, 73 (1967).

[34] D. N. Spergel et. al., Astrophys. J. Suppl. Ser. 148, 175
(2003).

[35] D. S. Salopek and J. R. Bond, Phys. Rev. D 43, 1005 (1991).

[36] S. Mollerach, S. Matarrese, A. Ortolan, and F. Lucchin, Phys. Rev. D 44, 1670 (1991).

[37] G. I. Rigopoulos and E. P. S. Shellard, astro-ph/0405185

[38] G. I. Rigopoulos, E. P. S. Shellard, and B. J. W. van Tent, astro-ph/0504508

[39] A. Linde, JHEP 0111, 052 (2001).

[40] L. Boubekeur and D. H. Lyth, hep-ph/0502047

[41] D. S. Salopek, Phys. Rev. D 45, 1139 (1992).

[42] N. Bartolo, S. Matarrese, and A. Riotto, Phys. Rev. D 65, 103505 (2002).

[43] F. Bernardeau and J. P. Uzan, Phys. Rev. D 67, 121301(R) (2003).

[44] F. Bernardeau and J. P. Uzan, Phys. Rev. D 66, 103506 (2002).

[45] K. Enqvist and A. Väihkönen, JCAP 0409, 006 (2004).

[46] K. Enqvist, A. Jokinen, A. Mazumdar, T. Multamäki, and A. Väihkönen, hep-th/0502185.

[47] J. H. Traschen and R. H. Brandenberger, Phys. Rev. D 42, 2491 (1990).

[48] L. Kofman, A. Linde and A. A. Starobinsky, Phys. Rev. Lett. 73, 3195 (1994).

[49] B. A. Bassett, D. I. Kaiser, and R. Maartens, Phys. Lett. B 455, 84 (1999).

[50] B. A. Bassett, F. Tamburini, D. I. Kaiser, and R. Maartens, Nucl. Phys. B 561, 188 (1999).

[51] K. Enqvist, A. Jokinen, A. Mazumdar, T. Multamäki, and A. Väihkönen, Phys. Rev. Lett. 94, 161301 (2005).

[52] K. Enqvist, A. Jokinen, A. Mazumdar, T. Multamäki, and A. Väihkönen, JCAP 0503, 010 (2005).

[53] M. Bastero-Gil, V. Di Clemente, and S. F. King, Phys. Rev. D 67, 103516 (2003).

[54] S. Antusch, M. Bastero-Gil, S. F. King, and Q. Shafi, Phys. Rev. D 71, 083519 (2005).

[55] E. W. Kolb, A. Riotto, and A. Vallinotto, Phys. Rev. D 71, 043513 (2005).

[56] D. H. Lyth and D. Wands, Phys. Lett. B 524, 5 (2002).

[57] D. H. Lyth, C. Ungarelli, and D. Wands, Phys. Rev. D 67, 023503 (2003).

[58] N. Bartolo, S. Matarrese, and A. Riotto, Phys. Rev. D 69, 043503 (2004).

[59] D. H. Lyth, Phys. Lett. B 579, 239 (2004).

[60] G. Dvali, A. Gruzinov, and M. Zaldarriaga, Phys. Rev. D 69, 023505 (2004).

[61] M. Zaldarriaga, Phys. Rev. D 69, 043508 (2004).

[62] G. Dvali, A. Gruzinov, and M. Zaldarriaga, Phys. Rev. D 69, 083505 (2004).

[63] F. Vernizzi, Phys. Rev. D 69, 083526 (2004).

[64] L. Verde and A. F. Heavens, Astrophys. J. 553, 14 (2001).

[65] W. Hu, Phys. Rev. D 64, 083005 (2001).

[66] NASA's Wilkinson Microwave Anisotropy Probe homepage: http://wmap.gsfc.nasa.gov/ 